\title{
TCF4 sequence variants and mRNA levels are associated with neurodevelopmental characteristics in psychotic disorders
}

\author{
KV Wirgenes ${ }^{1,2}$, IE Sønderby ${ }^{3}$, UK Haukvik ${ }^{1,4}$, M Mattingsdal $^{1}$, M Tesli $^{1,2}$, L Athanasiu $^{1,2}$, K Sundet $^{2,5}$, Jl Røssberg $^{1,2}$, AM Dale $^{6,7}$, \\ AA Brown ${ }^{1}$, I Agartz $^{1,4}$, I Melle ${ }^{1,2}$, S Djurovic ${ }^{1,2,3}$ and OA Andreassen ${ }^{1,2}$
}

TCF4 is involved in neurodevelopment, and intergenic and intronic variants in or close to the TCF4 gene have been associated with susceptibility to schizophrenia. However, the functional role of TCF4 at the level of gene expression and relationship to severity of core psychotic phenotypes are not known. TCF4 mRNA expression level in peripheral blood was determined in a large sample of patients with psychosis spectrum disorders $(n=596)$ and healthy controls $(n=385)$. The previously identified TCF4 risk variants (rs12966547 (G), rs9960767 (C), rs4309482 (A), rs2958182 (T) and rs17512836 (C)) were tested for association with characteristic psychosis phenotypes, including neurocognitive traits, psychotic symptoms and structural magnetic resonance imaging brain morphometric measures, using a linear regression model. Further, we explored the association of additional 59 single nucleotide polymorphisms (SNPs) covering the TCF4 gene to these phenotypes. The rs12966547 and rs4309482 risk variants were associated with poorer verbal fluency in the total sample. There were significant associations of other TCF4 SNPs with negative symptoms, verbal learning, executive functioning and age at onset in psychotic patients and brain abnormalities in total sample. The TCF4 mRNA expression level was significantly increased in psychosis patients compared with controls and positively correlated with positive- and negative-symptom levels. The increase in TCF4 mRNA expression level in psychosis patients and the association of TCF4 SNPs with core psychotic phenotypes across clinical, cognitive and brain morphological domains support that common TCF4 variants are involved in psychosis pathology, probably related to abnormal neurodevelopment.

Translational Psychiatry (2012) 2, e112; doi:10.1038/tp.2012.39; published online 8 May 2012

\section{Introduction}

Schizophrenia and bipolar disorder are characterized by psychotic symptoms, mood dysregulation and cognitive dysfunction, and are regarded as complex genetic disorders with mainly unknown underlying pathophysiology. Despite the high heritability, the genetic susceptibility factors are only partly understood. Large genome-wide association studies (GWAS) have provided the opportunity to identify new genes and genetic variation in new pathways. ${ }^{1}$ Using appropriate sample sizes, GWAS have identified several disease-associated gene variants, ${ }^{2-4}$ and in schizophrenia, these studies indicate the presence of neurodevelopmental and immunerelated mechanisms. ${ }^{4,5}$

One of the findings in schizophrenia is association to the gene transcription factor 4 (TCF4), located on chromosome 18q21, coding for a basic helix-loop-helix transcription factor. First, Stefansson et al. (2009) ${ }^{4}$ combined data from several GWAS and reported seven single nucleotide polymorphisms (SNPs) associated with schizophrenia at a genome-wide level, including rs9960767 located in an intron of TCF4 $\left(P=4.1 \times 10^{-9}\right)$. A study of Han Chinese patients replicated this association through another SNP (rs2958182, in high linkage disequilibrium (LD) with rs9960767). ${ }^{6}$ Rs4309482, situated intergenically downstream of TCF4 and upstream of CCDC68, was reported in another large GWAS. ${ }^{7}$ Finally, in recent mega-analyses, two new TCF4 SNPs (rs17512836 (intron 3 of TCF4) and rs12966547 (in high LD with rs4309482)) were identified and further supported TCF4 as a disease gene for schizophrenia. ${ }^{5}$ Interestingly, TCF4 has also been associated with bipolar disorder, ${ }^{8}$ which is in line with the growing evidence supporting overlapping genetic factors in schizophrenia and bipolar disorders, ${ }^{9,10}$ and corresponding to the overlapping clinical and neurocognitive features. ${ }^{11}$ It is possible that TCF4 risk loci confer risk for psychosis-related phenotypes across diagnostic boundaries.

TCF4 variations have been shown to be responsible for Pitt-Hopkins syndrome characterized by severe mental retardation, developmental delay, microcephaly, hyperventilation episodes and characteristic dysmorphisms

\footnotetext{
${ }^{1}$ Institute of Clinical Medicine, University of Oslo, Oslo, Norway; ${ }^{2}$ Division of Mental Health and Addiction, Oslo University Hospital- Ulleval, Oslo, Norway; ${ }^{3}$ Department of Medical Genetics, Oslo University Hospital-Ulleval, Oslo, Norway; ${ }^{4}$ Department of Psychiatric Research, Diakonhjemmet Hospital, Oslo, Norway; ${ }^{5}$ Department of Psychology, University of Oslo, Oslo, Norway; ${ }^{6}$ Department of Radiology, University of California, San Diego, La Jolla, CA, USA and ${ }^{7}$ Department of Neurosciences, University of California, San Diego, La Jolla, CA, USA

Correspondence: KV Wirgenes, Psychosis Research Section - TOP, Building 49, Division of Mental Health and Addiction, Oslo University Hospital - Ulleval, Kirkeveien 166, N-0407 Oslo, Norway.

E-mail: k.v.wirgenes@medisin.uio.no

Keywords: bipolar disorder; mRNA; neurodevelopment; psychotic phenotypes; schizophrenia; transcription factor 4

Received 13 Feburary 2012; revised 29 March 2012; accepted 5 April 2012
} 
(http://omim.org). ${ }^{12,13}$ Haploinsufficiency caused by deletions and nonsense mutations is the presumed molecular mechanism, but missense mutations are also seen. ${ }^{12}$ Thus, genetic variation in TCF4 may affect cognition and several neuropsychiatric phenotypes in both psychiatric patients and controls. $^{13}$

Cell and animal studies have indicated an important role of TCF4 in neuronal development. It is highly expressed in the embryonic central nervous system and schlerotomal component of the somites and the adult brain ${ }^{14}$ and severe disruption in pontine nuclei development has been reported in $\mathrm{TCF}^{-/-}$ mice. ${ }^{15}$ In addition, cognitive impairments and deficits in pre-pulse inhibition were found in mice overexpressing TCF4 in the forebrain. ${ }^{16}$ These studies indicate that TCF4 may affect a range of brain-related phenotypes, but with regard to psychosis, TCF4 has mainly been investigated in relation to case-control status.

The neurodevelopmental hypothesis for schizophrenia is supported by the observations of increased occurrence of obstetric complications, reduced premorbid function in children who later develop schizophrenia, cognitive dysfunction, positive and negative symptoms and reduced cortical thickness and enlarged ventricles in the early phases of the disease. ${ }^{17-19}$ It is possible that common variants in genes controlling neurodevelopment, such as TCF4, confer risk for phenotypes related to neurodevelopmental aspects of psychosis.

The object of the current study was to further elucidate the role of TCF4 in psychosis pathology, by testing the hypothesis that TCF4 variants are associated with psychosis phenotypes related to abnormal neurodevelopment. We determined TCF4 mRNA expression level in patients with psychotic disorders and controls. Next, we tested whether previously identified schizophrenia risk variants (rs12966547, rs2958182, rs9960767, rs4309482 and rs17512836) ${ }^{4-7}$ in TCF4 were associated with neurodevelopmental phenotypes of psychotic disorders (early age at onset, positive and negative symptoms, cognitive dysfunction and brain magnetic resonance imaging (MRI) morphometric measures) independent of diagnostic boundaries using a large well-described sample of patients with schizophrenia and bipolar disorder and healthy controls. Further, we aimed to explore any specific association with the schizophrenia diagnostic group. Finally, we did an exploratory association analysis with other TCF4 variants.

\section{Methods and materials}

Sample. The participants are part of a larger Norwegian study sample (Thematically Organized Psychosis (TOP) Study), which is a collaborative study involving the University of Oslo and all the Hospitals in the Oslo region, funded by the University, Regional Health Authorities and the Research Council of Norway.

A total of 596 patients with psychotic disorders or affective psychosis according to DSM-IV and 385 healthy controls were included. The patients were divided into three groups: (1) schizophrenia spectrum disorders, referred to as schizophrenia in the following: encompassing schizophrenia $(n=225)$, schizoaffective disorder $(n=23)$ and schizophreniform disorder $(n=52)$, (2) bipolar spectrum disorder, referred to as bipolar disorder in the following: encompassing bipolar disorder type $1(n=131)$, bipolar disorder type $2(n=60)$, bipolar disorder not otherwise specified $(n=13)$ and major depressive disorder with psychosis $(n=19)$ and (3) psychosis classified as 'other psychosis', that is, non-schizophrenia or bipolar disorder $(n=73)$. Main inclusion criteria in addition to the mentioned DSM-IV disorders were age between 18 and 65 years, absence of mental retardation and no history of major brain trauma.

The group of healthy controls $(n=385)$ was from the same catchment area as the patient group and randomly selected from national statistical records (www.ssb.no). The healthy control sample was evaluated with a clinical interview about severe mental disorder symptoms and the Primary Care Evaluation of Mental Disorders (PRIME-MD). ${ }^{20}$ The subjects were excluded if they or any of their close relatives had a lifetime history of a severe psychiatric disorder (schizophrenia, bipolar disorder or major depression), if they had an unstable medical condition known to interfere with brain function, or substance abuse or dependency in the past 3 months. A detailed description of the sample has been reported elsewhere. ${ }^{21}$ Demographic and clinical data are presented in Table 1.

In the described sample, a total of 895 participants had both neurocognitive and genotype data, 542 had mRNA data and genotype data, 480 had both brain imaging and genotype data and a total of 717 had both mRNA and clinical data. Furthermore, a total of 300 patients with schizophrenia had clinical data and genotype data. For details, see Supplementary Tables S1 and S2 online.

The study was approved by The Norwegian ScientificEthical Committees and the Norwegian Data Protection Agency, and the biobank was approved by the Health Authorities. Written informed consent was given from all participants before inclusion.

Clinical characterization. Clinical assessment was carried out by trained physicians and clinical psychologists. A standardized protocol of operational instruments was used. $^{22}$ Diagnosis was based on the Structured Clinical Interview for DSM-IV Axis I disorders (SCID-I). ${ }^{23}$ Diagnostic reliability was found satisfactory, with overall agreement for DSM-IV diagnostic categories of $82 \%$ with $\kappa=0.77$ (95\% confidence interval: $0.60-0.94) .{ }^{22}$ In addition, information from follow-up visits was used to secure correct diagnoses. In order to specifically address core psychotic features, the following assessments were used: Current positive and negative psychiatric symptoms were assessed using the Positive and Negative Syndrome Scale (PANSS). ${ }^{24}$ Psychosocial functioning in patients was assessed with the Global Assessment of Functioning scale, split version. ${ }^{25}$ Age at onset was obtained from interview and/or hospital records. For the current analysis, phenotypes related to neurodevelopment (age at onset and positive and negative symptoms (PANSS positive and negative sum-score)) were selected. ${ }^{17}$ All subjects reported their alcohol and drug intake in the past 2 weeks before the interview. 


\begin{tabular}{|c|c|c|c|c|}
\hline & Schizophrenia $(\mathrm{n}=300)$ & Bipolar disorder $(n=223)$ & Other psychoses $(n=73)$ & Healthy controls $(\mathrm{n}=385)$ \\
\hline \multicolumn{5}{|l|}{ Demographics } \\
\hline Age, years ${ }^{b}$ & $32.6(9.7)$ & $36.0(12.1)$ & $33.0(10.3)$ & $34.6(10.0)$ \\
\hline Sex, $n$ (\% male $)$ & $161(53.7)$ & 85 (38.1) & $47(64.4)$ & $197(51.2)$ \\
\hline Age at onset, years ${ }^{c}$ & $27.2(8.6)$ & $28.3(11.1)$ & $26.1(14.6)$ & - \\
\hline \multicolumn{5}{|l|}{ Symptom ratings } \\
\hline PANSS total score & $64.2(16.9)$ & $46.3(10.7)$ & $55.8(14.8)$ & - \\
\hline GAF symptom & $41.3(11.3)$ & $57.0(11.7)$ & $48.5(13.6)$ & - \\
\hline GAF function & $42.5(10.3)$ & $54.6(12.2)$ & $50.4(14.1)$ & - \\
\hline Medication & $N(\%)$ & $N(\%)$ & $N(\%)$ & \\
\hline Antipsychotic & $268(89.3)$ & $113(50.6)$ & $54(74.0)$ & - \\
\hline Lithium & $7(2.3)$ & $34(15.2)$ & $0(0)$ & - \\
\hline Anticonvulsiva & $60(20.0)$ & $98(44.0)$ & $8(11.0)$ & - \\
\hline Antidepressants & 89 (29.7) & $84(37.6)$ & $26(35.6)$ & - \\
\hline Sedatives & $28(12.4)$ & $34(11.3)$ & $16(21.9)$ & - \\
\hline
\end{tabular}

Abbreviations: GAF, Global Assessment of Functioning; PANSS, Positive and Negative Syndrome Scale.

a Mean and standard deviation are reported unless otherwise specified. ${ }^{b}$ Age was defined as age at phenotype assessments. ${ }^{\mathrm{c}} \mathrm{Age}$ at onset was defined as age at first contact with the mental health service owing to a primary symptom.

Neuropsychological testing. All participants were tested with a clinically relevant neuropsychological battery. For the current analysis, cognitive tests that have revealed cognitive dysfunction in schizophrenia and bipolar disorder were selected. ${ }^{11}$ The test battery included the following tests: general cognitive function measured using the Wechsler Abbreviated Scale of Intelligence (WASI). ${ }^{26}$ Mental speed was evaluated by the Digit Symbol Coding Test (WAIS-III). ${ }^{27}$ Executive function was evaluated by the Inhibition and Inhibition/Switching on Color-Word Interference Test, and by Verbal Fluency Letter, Verbal Fluency Category and Category Switching from the Verbal Fluency Test (Delis Kaplan Executive Function System (D-KEFS)). ${ }^{28}$ Verbal memory was measured by the total learning score (trials A1-5) from the California Verbal Learning Test- Second edition (CVLT-II). ${ }^{29}$ Low scores equal poorer performance for all tests apart from the Color-Word Interference Test.

MR image acquisition and processing. A subsample of 480 participants underwent MRI scanning on the same 1.5T. scanner (Siemens Magnetom Sonata scanner, Siemens Medical Solutions, Erlangen, Germany) equipped with a standard head coil at the Oslo University Hospital, Oslo, Norway between 2003 and 2009. ${ }^{30}$ After a conventional 3-plane localizer, two sagittal T1-weighted magnetizationprepared rapid gradient echo volumes were acquired with the Siemens tfl3d1_ns pulse sequence $(T E=3.93 \mathrm{~ms}, \mathrm{TR}=$ $2730 \mathrm{~ms}, \mathrm{TI}=1000 \mathrm{~ms}$, flip angle $=7^{\circ} ; \mathrm{FOV}=24 \mathrm{~cm}$, voxel size $=1.33 \times 0.94 \times 1 \mathrm{~mm}^{3}$, number of partitions $=160$ ). Acquisition parameters were optimized for increased gray/ white matter image contrast. All brain scans were read by a specialist in neuroradiology and were found to be free from organic brain pathology (for example, tumors, bleeding, infarcts and obstructive hydrocephalus).

The image files in DICOM format were transferred to a Linux workstation for morphometric analysis. Images were corrected for non-linear warping caused by gradient coil nonlinearities, using tools developed through the Morphometry
Biomedical Informatics Research Network, ${ }^{31}$ and the two T1-weighted images were rigid body registered to each other (motion corrected). The FreeSurfer 3.0.2 software package (http://surfer.nmr.mgh.harvard.edu) was used to obtain measures of subcortical volumes ${ }^{32}$ and cortical area and thickness. ${ }^{33,34}$ Summary measures were generated and used as structural phenotypes. ${ }^{35}$ For more details, see Supplementary Information online.

We included seven morphometric summation measures ${ }^{35}$ shown to be abnormal in schizophrenia and bipolar disorders: ${ }^{30}$ frontal cortical area and thickness, temporal cortical area and thickness, hippocampal volume, cerebellar volume and total ventricular volume (comprising the lateral and inferior lateral, third and fourth ventricle). Based on the finding of microcephaly in patients with Pitt-Hopkins syndrome, we also investigated total brain volume.

Genotyping and SNP selection. The sample was genotyped at Expression Analysis (Durham, NC, USA) using the Affymetrix Genome-Wide Human SNP Array 6.0. (Affymetrix, Santa Clara, CA, USA). Quality control was performed using PLINK ${ }^{36}$ Details about genotyping and quality control procedures are provided in supplementary information. This resulted in a sample of 1377 individuals with suitable genotype data available; of the 909,622 markers on the array, 525,117 autosomal markers remained. The total genotyping rate in remaining individuals was 0.993 . We extracted SNPs from the TCF4 gene region based on UCSC coordinates $\pm 20 \mathrm{~kb} k b$ (chr 18, 52869562-53323185 bp (hg19)) leaving a total of 59 SNPs (see Supplementary Table S3 online). Five additional SNPs in or near the TCF4 gene were selected because of association with schizophrenia (rs12966547, rs2958182, rs9960767, rs4309482 and rs17512836). ${ }^{4-7}$ They were imputed by IMPUTE2 ${ }^{37}$ using 1000 genomes CEU sample as reference. LD blocks are defined by confidence bounds on the normalized measure of allelic association $\left(D^{\prime}\right)^{38}$ (see Supplementary Figure S1 online). 
RNA measures. Total RNA was isolated from blood using the Tempus 12-Port RNA Isolation kit (Applied Biosystems; Ambion, Austin, TX, USA) and quantified using the ND-1000 spectrophotometer (NanoDrop Technologies, Wilmington, DE, USA).

Quantitative real-time RT-PCR was performed by using the ABI Prism $7900 \mathrm{HT}$ sequence detection system (Applied Biosystems, Foster City, CA, USA). RNA (1 $\mu \mathrm{g})$ was reversetranscribed using a high-capacity cDNA RT kit (Applied Biosystems), and quantitative RT-PCR was performed using TaqMan Gene Expression Master Mix (Applied Biosystems). The Assays-on-Demand products purchased from Applied Biosystems contained Taqman minor groove binder probes (6-FAM dye-labeled) combined with the primers for the genes of interest: TCF4 (Hs00162613_m1). An Assay-on-Demand product for eukaryotic ACTB (ACTB: Hs99999903_m1) was used as endogenous control. Details about RNA quantification procedure are provided in supplementary information online.

Statistical analysis. Potential differences in TCF4 mRNA level between the diagnostic groups and control group were analyzed using an analysis of covariance in the PASW software package for Windows, version 18.0. (PASW, Chicago, IL). TCF4 mRNA level was entered as the dependent variable, diagnostic group as the independent variable. Age was significantly correlated with TCF4 mRNA level (Pearson $r=0.14, P<0.005)$, and was entered as a covariate in the analysis. Independent sample $t$-test showed no statistical effect of gender on TCF4 mRNA level (t $(714)=0.99, P>0.05)$. As psychotropic medication may affect the TCF4 mRNA level, a separate one-way analysis of variance was conducted to explore the effect of medication. The patient sample was divided into six non-overlapping groups based on their prescription (1: Antipsychotics; 2: Lithium; 3: Anticonvulsiva; 4: Antidepressants; 5: Combinational treatment of more than one type of psychotropic; 6: Unmedicated; see Supplementary Table S4 online). Partial correlation ( $r$ ) controlling for age was used to investigate how positive and negative symptoms related to TCF4 mRNA as a previously study had found a correlation between delusions and TCF4 mRNA. ${ }^{39}$

Associations between TCF4 SNPs and core psychotic phenotypes were tested through the additive model of linear regression in PLINK. ${ }^{36}$ The whole sample was investigated for association to cognitive measures, MRI and mRNA. Additionally, associations with psychosis symptoms and age at disease onset were investigated in the whole subset of patients. Each analysis controlled for gender, age at sampling and diagnostic group ('schizophrenia', 'bipolar', 'other psychosis' or control). The effect in the schizophrenia group only, controlling for age and gender, was also analyzed.

Our primary focus was the five SNPs (rs12966547, rs9960767, rs2958182, rs4309482 and rs17512836) implicated in schizophrenia. Firstly, we analyzed these SNPs with general cognitive function, mental speed, executive function and verbal memory, then PANSS and age at onset. Using the same linear model, we investigated association to the eight morphometric summation measures: frontal cortical area and thickness, temporal cortical area and thickness, hippocampal volume, cerebellar volume, total ventricular volume and total brain volume.
Correction for multiple testing. A Bonferroni correction method was used to correct for multiple testing across independent variables after taking the correlation between genotypes and phenotypes into account. LD blocks determined by confidence bounds on the normalized measure of allelic association $\left(D^{\prime}\right)$ define number of independent SNPs. ${ }^{38}$ Several of the 19 phenotypes in the current study are highly correlated (see correlation matrix, Supplementary Table S5 online) and subdivision into phenotype families is one method to account for this. ${ }^{40}$ The phenotype families in the current study are: (1) TCF4 mRNA, (2) positive and negative symptoms, (3) cognitive tests performance, and (4) brain morphological summary measures. Owing to evidence that rs12966547, rs9960767, rs2958182, rs4309482 and rs17512836 are associated with schizophrenia and our a priori hypothesis for an association between the risk SNPs and key phenotypes in psychosis, Bonferroni correction for four independent SNPs (see Supplementary Figure S1A online) and four independent phenotype families were performed and significance level was set to $P<0.0031$ in these analyses. The 59 TCF4 SNPs were tested for the same core psychosis phenotypes mentioned above. The $P$-values for the SNP-phenotype analyses were corrected for multiple testing with Bonferroni correction for 14 independent SNPs (see Supplementary Figure S1B online) and four independent phenotype families and the significance level was set to $P<0.00089$.

\section{Results}

TCF4 mRNA expression. We measured TCF4 mRNA expression in the blood of patients with schizophrenia, bipolar disorder, other psychoses and controls. There was a significant difference in TCF4 mRNA expression level between the groups after correcting for age $(F(3,711)=8.23$, $P=2.2 \times 10^{-5}$ ), with a higher relative expression in patient groups compared with controls (Figure 1). Post hoc Tukey tests revealed significantly higher relative mRNA level in all individual patient groups in comparison to controls: schizophrenia $\left(2.22 \%, P=9.0 \times 10^{-5}\right)$, bipolar disorder $(1.78 \%$, $P=0.005$ ) and other psychoses (1.87\%, $P=0.049$ ) (see Supplementary Table S6 online). No significant difference between the individual patient groups was observed (see Supplementary Table S6 online). Medication status (see Supplementary Table S4 online) was significantly associated with TCF4 mRNA level $(\mathrm{F}(5,499)=2.7, P=0.020)$. Post hoc Tukey tests did not show any significant mRNA differences between the medication groups. Comparison of mRNA level in all unmedicated patients $(n=74)$ and controls $(n=212)$ revealed a trend of higher mRNA level in patients $(\mathrm{t}(284)=$ 1.37, $P=0.17)$. Next, positive symptoms $(r=0.10, P=$ $0.027)$ and negative symptoms $(r=0.13, P=0.004)$ were positively correlated with TCF4 mRNA level in total sample and negative symptoms $(r=0.16, P=0.016)$ in schizophrenia sample (see Supplementary Table S7 online).

To investigate if the mRNA differences could be associated with TCF4 gene variants, we tested SNPs previously associated with schizophrenia: rs12966547, rs9960767, rs2958182, rs4309482 and rs17512836 (the 'schizophrenia 
SNP analysis'). We also tested a set of 59 additional SNPs in the TCF4 gene, which we called the 'exploratory analysis'. Each analysis was performed in the entire sample as well as in schizophrenia patients separately. Nominal significant associations between TCF4 SNP variants and TCF4 mRNA expression levels were found (see Supplementary Table S7 online) but did not remain significant after correcting for multiple testing. No significant eQTLs for TCF4 mRNA were found in our data (data not shown).

TCF4 SNPs and clinical characteristics. Nominal significant results from the 'schizophrenia SNP analysis' are presented in Table 2 and results remaining significant after correction for multiple testing from the 'exploratory analysis' are presented in Table 3 and Figure 2. To investi-

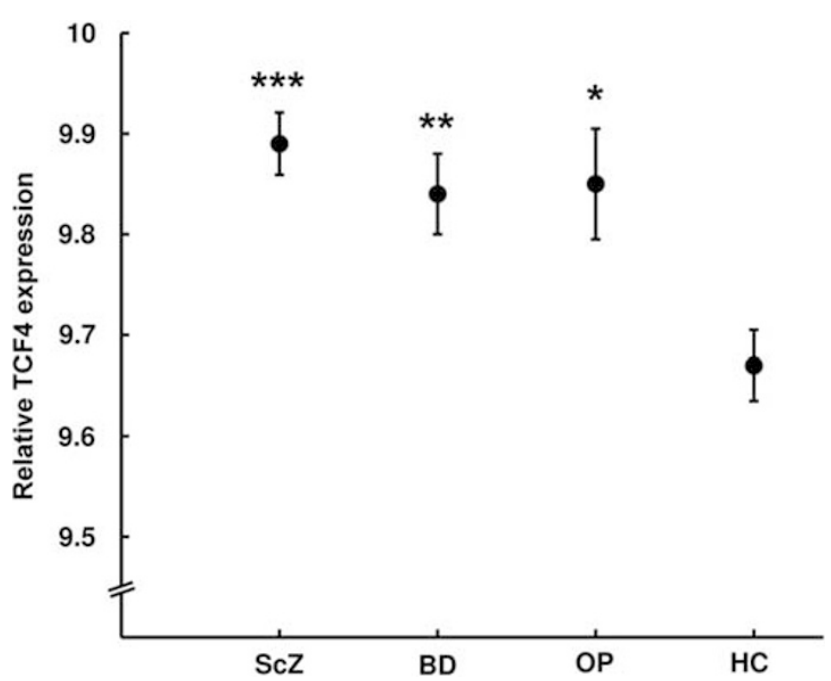

Figure 1 Relative TCF4 mRNA expression between the diagnostic groups The figure shows significantly lower TCF4 mRNA expression in healthy controls (HC, $n=212$ ) compared with ${ }^{* * *}$ schizophrenia (ScZ, $n=232, P=8.6 \times 10^{-5}$ ), ${ }^{*}$ bipolar disorder (BD, $\left.n=200, P=0.005\right)$ and *other psychoses (OP, $n=73$, $P=0.049)$. Mean values with \pm one standard error are presented. gate if common TCF4 gene variants are associated with sub-phenotypes of psychosis characteristic of abnormal neurodevelopment, we tested PANSS positive and negative sum scores and age at onset. In the 'exploratory analysis', minor alleles of rs2958188 and major allele of rs9636107 in each of their LD block were associated with younger age at onset in the total patient sample, and minor alleles of eleven SNPs (rs1261086, rs1261073, rs1261076, rs1942265, rs7241077, rs1893431, rs4800988, rs1261134, rs1788027, rs1660242 and rs1788014) from sequential LD blocks were associated with less negative symptoms in patients with schizophrenia (Table 3 ). There was no significant association with positive symptoms.

TCF4 SNPs and cognitive functions. We analyzed whether the TCF4 SNPs were associated with cognitive dysfunction, another sub-phenotype of psychosis characteristic of abnormal neurodevelopment. In the 'schizophrenia SNP analysis', risk alleles of the tightly linked rs12966547 and rs4309482 were associated with poorer performance on Verbal Fluency in total sample after correction for multiple testing (Table 2). In addition, risk alleles of rs12966547 and s4309482 were associated with poorer verbal fluency in patients with schizophrenia and risk allele of rs2958182 was associated with better performance on Color-Word Interference test (inhibition) in patients with schizophrenia, but these findings did not survive correction for multiple testing.

In the 'exploratory analysis', the minor alleles of rs 1377243 and rs2958188 and major alleles of rs41396445 and rs10871582 in two LD blocks were associated with poorer performance on verbal memory (CVLT) in the schizophrenia sample (Table 3).

TCF4 SNPs and structural brain MRI measures. We tested if TCF4 SNPs were associated with MRI morphometric summary measures in brain regions found to be abnormal in psychosis. In the 'schizophrenia SNP analysis', the risk alleles of rs12966547 and s4309482 were associated with larger ventricular volumes and the risk allele of rs9960767 was associated with a larger hippocampus and smaller

Table 2 Results from the 'schizophrenia SNPs' and phenotypes analyses

\begin{tabular}{|c|c|c|c|c|c|c|c|c|c|c|c|c|}
\hline SNP & $\mathbf{R}^{2^{a}}$ & Localisation & $\begin{array}{l}\text { Minor } \\
\text { allele }\end{array}$ & MAF & $\begin{array}{l}\text { Risk } \\
\text { allele }\end{array}$ & Phenotype & Sample & $\mathbf{N}$ & Beta $^{\mathbf{b}}$ & $S E$ & $\boldsymbol{P}$ & Corr \\
\hline rs12966547 & 0.998 & 52752017 & A & 0.436 & $\mathrm{G}^{\mathrm{d}}$ & $\begin{array}{l}\text { Verbal fluency } \\
\text { Verbal fluency } \\
\text { Ventricular volume }\end{array}$ & $\begin{array}{l}\text { All } \\
\text { Schizophrenia } \\
\text { All }\end{array}$ & $\begin{array}{l}890 \\
227 \\
480\end{array}$ & $\begin{array}{l}-1.75 \\
-2.56 \\
1535\end{array}$ & $\begin{array}{l}0.55 \\
1.18 \\
696\end{array}$ & $\begin{array}{l}0.0014 \\
0.031 \\
0.028\end{array}$ & $\begin{array}{l}\mathbf{0 . 0 2 2} \\
0.50 \\
0.45\end{array}$ \\
\hline rs4309482 & 0.981 & 52750469 & $\mathrm{G}$ & 0.444 & $A^{f}$ & $\begin{array}{l}\text { Verbal fluency } \\
\text { Verbal fluency } \\
\text { Ventricular volume }\end{array}$ & $\begin{array}{l}\text { All } \\
\text { Schizophrenia } \\
\text { All }\end{array}$ & $\begin{array}{l}890 \\
227 \\
480\end{array}$ & $\begin{array}{l}-1.81 \\
-2.73 \\
1557\end{array}$ & $\begin{array}{l}0.55 \\
1.19 \\
699\end{array}$ & $\begin{array}{l}0.0010 \\
0.023 \\
0.026\end{array}$ & $\begin{array}{l}\mathbf{0 . 0 1 6} \\
0.37 \\
0.42\end{array}$ \\
\hline rs2958182 & 0.938 & 53149021 & $A$ & 0.373 & $\mathrm{~T}^{\mathrm{g}}$ & Inhibition $^{\mathrm{h}}$ & Schizophrenia & 227 & -5.37 & 2.13 & 0.012 & 0.19 \\
\hline rs9960767 & 0.949 & 53155002 & $\mathrm{C}$ & 0.056 & $\mathrm{C}^{\mathrm{i}}$ & $\begin{array}{l}\text { Hippocampal volume } \\
\text { Ventricular volume }\end{array}$ & $\begin{array}{l}\text { All } \\
\text { All }\end{array}$ & $\begin{array}{l}480 \\
480\end{array}$ & $\begin{array}{c}207 \\
-3811\end{array}$ & $\begin{array}{c}102 \\
1599\end{array}$ & $\begin{array}{l}0.043 \\
0.018\end{array}$ & $\begin{array}{l}0.69 \\
0.29\end{array}$ \\
\hline
\end{tabular}

Abbreviations: MAF, minor allele frequency; $N$, number; SE, standard error; SNP, single nucleotide polymorphism.

${ }^{a} R^{2}$ imputation score. ${ }^{b}$ Beta is estimated by increasing number of risk alleles. ${ }^{c} P$-values in bold remained significant after controlling for multiple testing across independent genotypes and phenotype families. ${ }^{\mathrm{d}}$ Ripke et al. ${ }^{\mathrm{e}}$ Verbal Fluency Letter from the Verbal Fluency Test (Delis Kaplan Executive Function System (D-KEFS)). ${ }^{f}$ Steinberg et al. ${ }^{\mathrm{g}} \mathrm{Li}$ et al. ${ }^{\mathrm{h}}$ Inhibition from the Color-Word Interference Test (Delis Kaplan Executive Function System (D-KEFS)). 'Stefansson et al. Nominal significant results $(P<0.05)$ from the linear regression analysis of schizophrenia SNPs, correcting for diagnosis and gender. 
Table 3 Results of the 'Explorative Analysis' of TCF4 SNPs and phenotypes

\begin{tabular}{|c|c|c|c|c|c|c|c|c|c|c|c|}
\hline $\begin{array}{l}\text { LD } \\
\text { block }\end{array}$ & SNP & $\begin{array}{l}\text { Minor } \\
\text { allele }\end{array}$ & Phenotype & Sample & $N$ & $\operatorname{Mean}\left(\right.$ s.d.) ${ }^{\mathrm{a}}$ & $\operatorname{Mean}\left(\right.$ s.d.) ${ }^{\mathrm{b}}$ & $\operatorname{Mean}\left(\right.$ s.d.) ${ }^{\mathrm{c}}$ & $\begin{array}{l}\text { Effect } \\
\text { size }^{\mathrm{d}}\end{array}$ & $\boldsymbol{P}$ & Corr ${ }^{\mathrm{e}}$ \\
\hline 1 & rs1261086 & C & Negative symptoms & Scz & 282 & $13.51(6.10)$ & $15.51(6.67)$ & $16.94(6.67)$ & -0.21 & 0.00038 & 0.021 \\
\hline - & rs 1261073 & $\mathrm{G}$ & Negative symptoms & Scz & 281 & $13.39(6.26)$ & $15.06(5.91)$ & $17.26(7.45)$ & -0.23 & 0.00011 & 0.0062 \\
\hline 2 & rs 1261076 & $\mathrm{C}$ & Negative symptoms & Scz & 282 & $13.69(6.03)$ & $15.22(6.20)$ & $17.91(7.36)$ & -0.24 & 0.000039 & 0.0022 \\
\hline 2 & rs1942265 & $\mathrm{G}$ & Negative symptoms & Scz & 282 & $13.23(5.94)$ & $14.86(6.02)$ & $16.85(7.15)$ & -0.20 & 0.00059 & 0.033 \\
\hline 2 & rs7241077 & A & Negative symptoms & $\mathrm{Scz}$ & 282 & $13.23(5.94)$ & $14.86(6.02)$ & $16.85(7.15)$ & -0.20 & 0.00059 & 0.033 \\
\hline 2 & rs1893431 & $A$ & Negative symptoms & Scz & 282 & $13.47(6.31)$ & $15.02(5.09)$ & $17.26(7.41)$ & -0.22 & 0.00017 & 0.0095 \\
\hline 2 & rs4800988 & A & Negative symptoms & $\mathrm{Scz}$ & 282 & $13.23(5.94)$ & $14.86(6.02)$ & $16.85(7.15)$ & -0.20 & 0.00059 & 0.033 \\
\hline 2 & rs1261134 & $\mathrm{T}$ & Negative symptoms & Scz & 282 & $13.72(6.30)$ & $14.81(5.85)$ & $17.33(7.37)$ & -0.22 & 0.00011 & 0.0062 \\
\hline 2 & rs 1788027 & $\mathrm{~T}$ & Negative symptoms & $\mathrm{Scz}$ & 275 & $13.89(6.14)$ & $14.87(6.14)$ & $18.05(7.22)$ & -0.23 & 0.000077 & 0.0043 \\
\hline - & rs1660242 & $\mathrm{T}$ & Negative symptoms & $\mathrm{Scz}$ & 281 & $14.05(5.97)$ & $14.85(6.39)$ & $17.17(7.03)$ & -0.21 & 0.00045 & 0.025 \\
\hline 3 & rs1788014 & $G$ & Negative symptoms & Scz & 282 & $13.51(6.10)$ & $15.51(6.67)$ & $16.94(6.67)$ & -0.21 & 0.00038 & 0.021 \\
\hline 5 & rs1377243 & $\mathrm{T}$ & $\begin{array}{l}\text { California verbal } \\
\text { learning }\end{array}$ & Scz & 227 & $11.15(2.84)$ & $11.81(2.58)$ & $12.83(2.59)$ & -0.23 & 0.00044 & 0.025 \\
\hline \multirow[t]{2}{*}{6} & rs2958188 & $\mathrm{T}$ & Age at onset & Patients & 594 & $25.29(8.49)$ & $27.38(9.74)$ & $29.19(10.47)$ & -0.14 & 0.00079 & 0.044 \\
\hline & & & $\begin{array}{l}\text { California verbal } \\
\text { learning }\end{array}$ & Scz & 227 & $11.00(2.73)$ & $11.94(2.58)$ & $12.82(2.59)$ & -0.25 & 0.00014 & 0.0078 \\
\hline - & rs9636107 & $\mathrm{G}$ & Age at onset & Patients & 591 & $29.06(11.01)$ & $28.07(9.65)$ & $25.51(8.76)$ & 0.14 & 0.00049 & 0.027 \\
\hline \multirow[t]{2}{*}{9} & rs41396445 & $A$ & $\begin{array}{l}\text { Temporal cortical } \\
\text { area }\end{array}$ & All & 480 & $41660(4050)$ & 42480 (4231) & $43380(4511)$ & -0.12 & 0.00083 & 0.046 \\
\hline & & & $\begin{array}{l}\text { California verbal } \\
\text { learning }\end{array}$ & Scz & 227 & 12.95 (2.39) & $12.40(2.72)$ & $11.32(2.61)$ & 0.22 & 0.00081 & 0.045 \\
\hline 9 & rs682245 & $\mathrm{T}$ & Cerebellar volume & Scz & 106 & $146700(13250)$ & $135600(13020)$ & $132500(16150)$ & 0.28 & 0.00074 & 0.041 \\
\hline \multirow[t]{2}{*}{9} & rs17514242 & $\mathrm{G}$ & Temporal cortical area & All & 480 & 41440 (3966) & $42490(4274)$ & 43360 (4458) & -0.13 & 0.00053 & 0.030 \\
\hline & & & Brain volume & All & 480 & $1150000(121700)$ & $1170000(141900)$ & $1203000(134500)$ & -0.12 & 0.00067 & 0.038 \\
\hline 9 & rs12458118 & $A$ & Cerebellar volume & Scz & 106 & $146700(13250)$ & $135600(13020)$ & $132500(16150)$ & 0.28 & 0.00074 & 0.041 \\
\hline 9 & rs9951280 & $G$ & Cerebellar volume & Scz & 106 & $146700(13250)$ & $135600(13020)$ & $132500(16150)$ & 0.28 & 0.00074 & 0.041 \\
\hline \multirow[t]{3}{*}{9} & rs10871582 & $A$ & Temporal cortical area & All & 480 & 41520 (3982) & 42450 (4279) & $43370(4450)$ & -0.13 & 0.00056 & 0.031 \\
\hline & & & Brain volume & All & 480 & $1153000(122400)$ & $1169000(142200)$ & $1203000(134200)$ & -0.12 & 0.00083 & 0.046 \\
\hline & & & $\begin{array}{l}\text { California verbal } \\
\text { learning }\end{array}$ & Scz & 227 & $13.00(2.33)$ & $12.42(2.72)$ & $11.36(2.62)$ & 0.23 & 0.00083 & 0.046 \\
\hline
\end{tabular}

Abbreviations: LD, linkage disequilibrium; $N$, number; $P$, uncorrected $P$-value; s.d., standard deviation; SNP, single nucleotide polymorphism.

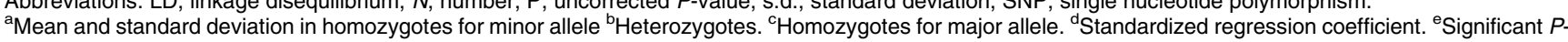
values after controlling for multiple testing across independent genotypes and phenotype families. Of note, six remained significant after controlling for all the 19 phenotypes. ${ }^{f}$ California Verbal Learning test- Second edition (CVLT-II), total learning score (trial A1-5). Scz: Schizophrenia group. Patients: all patients. All: total sample with patients and controls. Results surviving correction for multiple testing in the linear regression analysis of TCF4 SNPs and phenotypes, correcting for diagnosis and gender.

ventricles, but these findings did not remain significant after correction for multiple testing. The 'exploratory analysis' showed that the minor allele of three SNPs (rs41396445, rs17514242 and rs10871582) in the same LD block were associated with reduced temporal cortical area in total sample. Out of these, minor alleles of two SNPs (rs17514242 and rs10871582) were associated with reduced total brain volume in total sample. The major alleles of three SNPs (rs682245, rs12458118 and rs9951280) in the same LD block were associated with decreased cerebellar volume in patients with schizophrenia (Table 3).

\section{Discussion}

The main findings of the present study were upregulated TCF4 expression level in all psychotic diagnostic groups and that TCF4 risk variants associate with poorer verbal fluency in total sample. The exploratory analysis revealed that TCF4 variants associated with negative symptoms, cognitive dysfunction and cerebellar volume in the schizophrenia sample, age at onset in total patient sample and variants correlating with temporal cortical area and brain volume in total sample. This further implicates TCF4 in psychosis pathophysiology, and indicates a specific role in the development of clinical phenotypes related to aberrant neurodevelopment. To our knowledge, this is the first study on known TCF4 risk variants of schizophrenia and hitherto uncharacterized variants targeting psychosis phenotypes at different levels, including the peripheral TCF4 mRNA.

The analysis of known schizophrenia TCF4 risk variants revealed that the two linked rs12966547 and rs4309482 are associated with poorer executive function in the form of verbal fluency. Interestingly, reduced cognitive functioning has been associated with enlarged ventricular volume, ${ }^{41}$ corroborating our finding that the same risk variants, rs12966547 and rs4309482, were associated with larger ventricular volume even though this did not survive correction for multiple testing. Enlarged ventricular volume is one of the most consistent findings in schizophrenia, ${ }^{42}$ also found in bipolar disorder ${ }^{43}$ and Pitt-Hopkins syndrome. ${ }^{44}$ This indicates that these TCF4 risk SNPs might, at least partially, cause this common subphenotype in schizophrenia. ${ }^{41}$ The consistent pattern of effect across different phenotypes strengthens the findings.

The pattern of associations in the non-schizophrenia risk SNP analysis corroborates a relationship between TCF4 and changes in brain morphology. Several SNPs in one haploblock were associated with cerebellar volume, temporal cortical area or brain volume. The Allan Brain Atlas shows TCF4 expression in several parts of the brain, most pronounced in hippocampus and cerebellum (http:// www.brain-map.org). This is consistent with a role of TCF4 in early neurodevelopment ${ }^{45}$ and embryogenesis ${ }^{46}$ previously shown in animal models as well as concordant with microcephaly as a core characteristic of Pitt-Hopkins syndrome, ${ }^{44}$ 


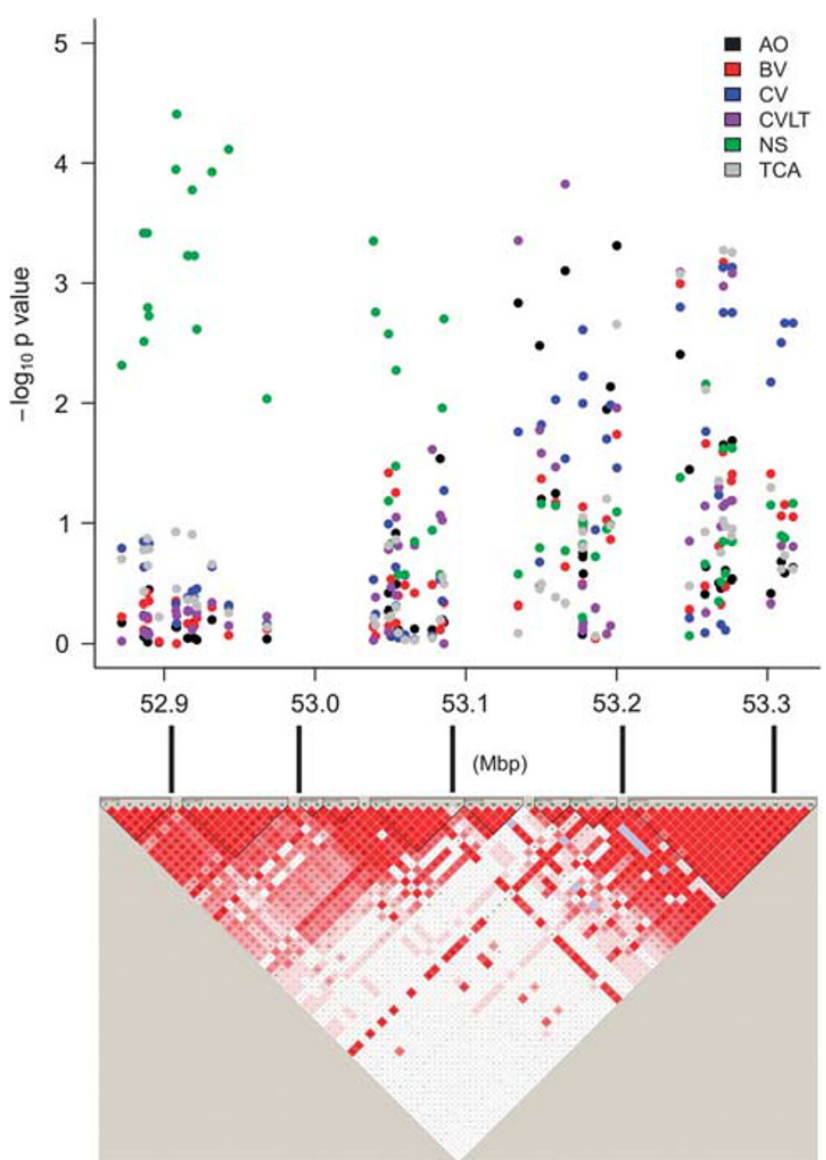

Figure 2 A TCF4 linkage disequilibrium map and a Manhattan plot with negative $\log P$-values (uncorrected) from the exploratory single nucleotide polymorphism-phenotype analysis. Only phenotypes remaining significant after multiple testing is illustrated. $\mathrm{AO}$, age at onset in total patient sample; $\mathrm{BV}$, brain volume in total sample; CV, cerebellar volume in schizophrenia sample; CVLT, California Verbal Learning test in schizophrenia sample; Mbp, mega base pair; NS, negative symptoms in schizophrenia sample; TCA, temporal cortical area in total sample.

as common variants in CDK5RAP2 and MCPH1 are associated with reduced brain volume and cortical area, ${ }^{47}$ while mutations cause primary microcephaly. ${ }^{48}$

Psychotic disorders are a heterogenous group of diseases and division into subgroups based on symptomatology might be desirable. The consistent effect of the TCF4 SNPs of several sequential haploblocks on negative symptoms in schizophrenia patients might be a path to define a subgroup of patients with more pronounced negative symptoms as previously suggested. ${ }^{49,50}$ Interestingly, the variants that affect negative symptoms are situated in the exon-dense region of the gene in which Pitt-Hopkins syndrome-causing mutations (exons 9-19) are identified. ${ }^{51}$ Furthermore, we found that one SNP was associated with both an earlier age at onset and worse verbal learning. Cognitive decline and early age at onset are predictors of poor prognosis in schizophrenia. $^{52}$ The pattern of clinical symptoms and cognitive dysfunction resembles what has been regarded as neurodevelopmental characteristics, and is consistent with an effect of TCF4 on abnormal neurodevelopment. The consistent pattern of effect across different SNPs on independent clinical, cognitive and brain imaging phenotypes suggests that the current findings are not due to chance.

TCF4 mRNA levels were significantly higher in the patient groups compared with controls. Measurements in the blood allow for larger sample sizes and better standardization of technical procedures, but it might not reflect mRNA levels in the brain. It has previously been reported that the availability of an accessible tissue, such as blood, with similar gene expression to a more inaccessible tissue, such as brain, may advance research in neuropsychiatric disorders. ${ }^{53}$ Sullivan et al. $^{53}$ reported that, on a transcriptome level, whole blood shares significant gene expression similarities with brain tissue. Thus, even though it was not perfectly correlated, gene expression in blood might be a useful surrogate marker for gene expression in the central nervous system. ${ }^{53}$ Our observation of increased level of TCF4 mRNA in patients with schizophrenia is consistent with increased cerebellar expression of TCF4 in schizophrenia patients shown in a genomic convergence analysis on post-mortem cerebellar cortices, ${ }^{54}$ indicating that in the case of TCF4, blood and brain expression might correlate. Microarrays of gene expression in blood is a practical method to use in psychotic patients and the technique has become increasingly accepted for studying biomarkers for neuropsychiatric diseases. ${ }^{55-63}$ For example, gene expression in peripheral blood mononuclear cells in patients with multiple sclerosis was correlated to disease activity. ${ }^{64}$ This is in line with our finding of a correlation between mRNA level and severity of positive and negative symptoms in patients with schizophrenia.

The result of increased TCF4 mRNA in psychotic patients should be interpreted with caution as an effect on mRNA levels of prescribed psychotropic medications cannot be excluded. We did find an overall effect of medication on mRNA level in patients, with the highest level in patients on antipsychotics or treated with several types of psychotropics, even though it did not survive correction for multiple testing. Thus, the observed effect might be a mirror of medication or reflect a more severe form of psychosis in patients on regular medical treatment. The trend of higher TCF4 mRNA level in unmedicated patients compared with controls does corroborate an effect regardless of medication, though. Another small-size (41 patients) study observed less TCF4 expression in blood from patients with higher rates of delusions. ${ }^{39}$ TCF4 mRNA level was positively correlated with positive and negative symptoms in our sample. This is in line with our finding of several SNPs associated with negative symptoms, which might reflect a difference in TCF4 expression in patients with high level of positive and negative symptoms and suggests that TCF4 variants may contribute to more pronounced symptoms. The reason for the apparent discrepancy between Kurian et al. and our findings might reflect different assumptions. While they compared TCF4 mRNA level in patients with schizophrenia with high level of delusions and patients with no delusions, our sample is generally in a more chronic phase of disease. Further, our sample is much larger and more heterogeneous and includes bipolar disorder and other psychoses, while they include different ethnicities. Therefore, before TCF4 mRNA can be used as a biomarker for psychosis, any putative effect of the level of positive and 
negative symptoms and medication on TCF4 mRNA level needs further investigation.

Two recent studies have found allele-specific expression of TCF4 in post-mortem human brains. ${ }^{65,66}$ No significant association between mRNA level and the common TCF4 polymorphisms was found in our data after correction for multiple testing, but there were nominal associations. The lack of significant support for cis-regulatory effect could be due to Type II error, as there are many factors that affect expression levels and may mask the effect. It may also be due to differences in eQTLs in blood and brain tissues. Given the observed small differences in transcript levels and the deleteriousness of hemizygosity for TCF4 observed in PittHopkins syndrome, it seems reasonable that evolutionary constraint on variants that alter expression levels might exist. Another option is that the locus only exerts its regulatory effect in a developmental context, or is organ specific. The gene expression in uteri and postnatal life has recently been examined, and the brain TCF4 mRNA level is highest in the early brain development in uteri, decreases in childhood and stabilizes in early adulthood. ${ }^{67}$ Thus, epigenetic factors from early life may have a continued effect on expression ${ }^{68}$ and together with a decrease in variation explain the lack of association between GWAS SNPs and TCF4 mRNA level in adults. ${ }^{67}$ It is also possible that none of the current TCF SNPs tagged the eQTLs.

Taken together, these results support earlier findings of TCF4 as an important gene in psychosis etiology, and suggest that TCF4 variants may lead to a subgroup of psychosis with clinical, cognitive and brain structural abnormalities related to neurodevelopmental mechanisms.

\section{Conflict of interest}

The authors declare no conflict of interest.

Acknowledgements. We thank all the participants for their contributions and the TOP study group for participation in data collection. This study was supported by grants to the TOP study group from the University of Oslo, the Research Council of Norway (167153/V50, 163070/v50), and the South-East Norway Health Authority (2010-074).

1. Cichon S, Craddock N, Daly M, Faraone SV, Gejman PV, Kelsoe J et al. Genomewide association studies: history, rationale, and prospects for psychiatric disorders. Am J Psychiatry 2009; 166: 540-556.

2. Sklar P, Ripke S, Scott LJ, Andreassen OA, Cichon S, Craddock N et al. Large-scale genome-wide association analysis of bipolar disorder identifies a new susceptibility locus near ODZ4. Nat Genet 2011; 43: 977-983.

3. Purcell SM, Wray NR, Stone JL, Visscher PM, O'Donovan MC, Sullivan PF et al. Common polygenic variation contributes to risk of schizophrenia and bipolar disorder. Nature 2009; 460: 748-752

4. Stefansson H, Ophoff RA, Steinberg S, Andreassen OA, Cichon S, Rujescu D et al. Common variants conferring risk of schizophrenia. Nature 2009; 460: 744-747.

5. Ripke S, Sanders AR, Kendler KS, Levinson DF, Sklar P, Holmans PA et al. Genome-wide association study identifies five new schizophrenia loci. Nat Genet 2011; 43: 969-976.

6. Li T, Li Z, Chen P, Zhao Q, Wang T, Huang $\mathrm{K}$ et al. Common variants in major histocompatibility complex region and TCF4 gene are significantly associated with schizophrenia in Han Chinese. Biol Psychiatry 2010; 68: 671-673.

7. Steinberg S, de JS, Andreassen OA, Werge T, Borglum AD, Mors $O$ et al. Common variants at VRK2 and TCF4 conferring risk of schizophrenia. Hum Mol Genet 2011; 20: 4076-4081.

8. Del-Favero J, Gestel SV, Borglum AD, Muir W, Ewald H, Mors $\mathrm{O}$ et al. European combined analysis of the CTG18.1 and the ERDA1 CAG/CTG repeats in bipolar disorder. Eur J Hum Genet 2002; 10: 276-280.
9. Lichtenstein P, Yip BH, Bjork C, Pawitan Y, Cannon TD, Sullivan PF et al. Common genetic determinants of schizophrenia and bipolar disorder in Swedish families: a populationbased study. Lancet 2009; 373: 234-239.

10. Williams HJ, Craddock N, Russo G, Hamshere ML, Moskvina V, Dwyer S et al. Most genome-wide significant susceptibility loci for schizophrenia and bipolar disorder reported to date cross-traditional diagnostic boundaries. Hum Mol Genet 2011; 20: 387-391.

11. Simonsen C, Sundet K, Vaskinn A, Birkenaes AB, Engh JA, Faerden A et al. Neurocognitive dysfunction in bipolar and schizophrenia spectrum disorders depends on history of psychosis rather than diagnostic group. Schizophr Bull 2011; 37: 73-83.

12. Giurgea I, Missirian C, Cacciagli P, Whalen S, Fredriksen T, Gaillon T et al. TCF4 deletions in Pitt-Hopkins Syndrome. Hum Mutat 2008; 29: E242-E251.

13. Blake DJ, Forrest M, Chapman RM, Tinsley CL, O'Donovan MC, Owen MJ. TCF4, schizophrenia, and Pitt-Hopkins Syndrome. Schizophr Bull 2010; 36: 443-447.

14. de Pontual L, Mathieu Y, Golzio C, Rio M, Malan V, Boddaert N et al. Mutational, functional, and expression studies of the TCF4 gene in Pitt-Hopkins syndrome. Hum Mutat 2009; 30: $669-676$.

15. Flora A, Garcia JJ, Thaller C, Zoghbi HY. The E-protein Tcf4 interacts with Math1 to regulate differentiation of a specific subset of neuronal progenitors. Proc Natl Acad Sci USA 2007; 104: 15382-15387.

16. Brzozka MM, Radyushkin K, Wichert SP, Ehrenreich H, Rossner MJ. Cognitive and sensorimotor gating impairments in transgenic mice overexpressing the schizophrenia susceptibility gene Tcf4 in the brain. Biol Psychiatry 2010; 68: 33-40.

17. Fatemi SH, Folsom TD. The neurodevelopmental hypothesis of schizophrenia, revisited. Schizophr Bull 2009; 35: 528-548.

18. Arnold SE, Talbot K, Hahn CG. Neurodevelopment, neuroplasticity, and new genes for schizophrenia. Prog Brain Res 2005; 147: 319-345.

19. Harrison PJ, Weinberger DR. Schizophrenia genes, gene expression, and neuropathology: on the matter of their convergence. Mol Psychiatry 2005; 10: 40-68.

20. Spitzer RL, Williams JB, Kroenke K, Linzer M, deGruy III FV, Hahn SR et al. Utility of a new procedure for diagnosing mental disorders in primary care. The PRIME-MD 1000 study. JAMA 1994; 272: 1749-1756.

21. Simonsen C, Sundet K, Vaskinn A, Birkenaes AB, Engh JA, Faerden A et al. Neurocognitive dysfunction in bipolar and schizophrenia spectrum disorders depends on history of psychosis rather than diagnostic group. Schizophr Bull 2009; 37: 73-83.

22. Birkenaes AB, Opjordsmoen S, Brunborg C, Engh JA, Jonsdottir H, Ringen PA et al. The level of cardiovascular risk factors in bipolar disorder equals that of schizophrenia: a comparative study. J Clin Psychiatry 2007; 68: 917-923.

23. Spitzer RL, Williams JB, Gibbon M, First MB. The Structured Clinical Interview for DSM-IIIR (SCID). I: History, rationale, and description. Arch Gen Psychiatry 1992; 49: 624-629.

24. Kay SR, Fiszbein A, Opler LA. The positive and negative syndrome scale (PANSS) for schizophrenia. Schizophr Bull 1987; 13: 261-276.

25. Pedersen G, Hagtvet KA, Karterud S. Generalizability studies of the Global Assessment of Functioning-Split version. Compr Psychiatry 2007; 48: 88-94.

26. Wechsler D, Wycherley RJ, Benjamin L. Wechsler Memory Scale: WMS-III. Stockholm, Sweden: Pearson Assessment, 2008.

27. Wechsler D. Wechsler Adult Intelligence Scale: WAIS-III. Stockholm, Sweden: Pearson Assessment, 2003.

28. Delis DC, Kaplan E, Kramer JH. The Delis-Kaplan Executive Function System: D-KEFS Stockholm, Sweden: Pearson Assessment, 2005

29. Delis DC, Kramer JH, Kaplan E, Ober BA. California Verbal Learning Test: CVLT-II. Stockholm, Sweden: Pearson Assessment, 2004.

30. Rimol LM, Hartberg CB, Nesvag R, Fennema-Notestine C, Hagler Jr DJ, Pung CJ et al. Cortical thickness and subcortical volumes in schizophrenia and bipolar disorder. Biol Psychiatry 2010; 68: 41-50.

31. Fennema-Notestine C, Gamst AC, Quinn BT, Pacheco J, Jernigan TL, Thal $L$ et al. Feasibility of multi-site clinical structural neuroimaging studies of aging using legacy data. Neuroinformatics 2007; 5: 235-245.

32. Fischl B, Salat DH, Busa E, Albert M, Dieterich M, Haselgrove $C$ et al. Whole brain segmentation: automated labeling of neuroanatomical structures in the human brain. Neuron 2002; 33: 341-355.

33. Dale AM, Fischl B, Sereno MI. Cortical surface-based analysis. I. Segmentation and surface reconstruction. Neuroimage 1999; 9: 179-194.

34. Fischl B, Sereno MI, Dale AM. Cortical surface-based analysis. II: Inflation, flattening, and a surface-based coordinate system. Neuroimage 1999; 9: 195-207.

35. Agartz I, Brown AA, Rimol LM, Hartberg CB, Dale AM, Melle I et al. Common sequence variants in the major histocompatibility complex region associate with cerebral ventricular size in schizophrenia. Biol Psychiatry 2011; 70: 696-698.

36. Purcell S, Neale B, Todd-Brown K, Thomas L, Ferreira MA, Bender D et al. PLINK: a tool set for whole-genome association and population-based linkage analyses. Am J Hum Genet 2007; 81: 559-575.

37. Howie BN, Donnelly P, Marchini J. A flexible and accurate genotype imputation method for the next generation of genome-wide association studies. PLoS Genet 2009; 5: e1000529.

38. Gabriel SB, Schaffner SF, Nguyen H, Moore JM, Roy J, Blumenstiel B et al. The structure of haplotype blocks in the human genome. Science 2002; 296: 2225-2229.

39. Kurian SM, Le-Niculescu H, Patel SD, Bertram D, Davis J, Dike C et al. Identification of blood biomarkers for psychosis using convergent functional genomics. Mol Psychiatry 2011; 16: 37-58. 
40. Shaffer JP. Multiple hypothesis testing. Annu Rev Psychol 1995; 46: 561-584.

41. Antonova E, Sharma T, Morris R, Kumari V. The relationship between brain structure and neurocognition in schizophrenia: a selective review. Schizophr Res 2004; 70 117-145.

42. Shenton ME, Dickey CC, Frumin M, McCarley RW. A review of MRI findings in schizophrenia. Schizophr Res 2001; 49: 1-52.

43. Arnone D, Cavanagh J, Gerber D, Lawrie SM, Ebmeier KP, McIntosh AM. Magnetic resonance imaging studies in bipolar disorder and schizophrenia: meta-analysis. Br J Psychiatry 2009; 195: 194-201.

44. Amiel J, Rio M, de PL, Redon R, Malan V, Boddaert N et al. Mutations in TCF4, encoding a class I basic helix-loop-helix transcription factor, are responsible for Pitt-Hopkins syndrome a severe epileptic encephalopathy associated with autonomic dysfunction. Am J Hum Genet 2007; 80: 988-993.

45. Brinkmeier ML, Potok MA, Davis SW, Camper SA. TCF4 deficiency expands ventral diencephalon signaling and increases induction of pituitary progenitors. Dev Biol 2007; 311: 396-407.

46. Korinek V, Barker N, Willert K, Molenaar M, Roose J, Wagenaar G et al. Two members of the Tcf family implicated in Wnt/beta-catenin signaling during embryogenesis in the mouse. Mol Cell Biol 1998; 18: 1248-1256.

47. Rimol LM, Agartz I, Djurovic S, Brown AA, Roddey JC, Kahler AK et al. Sex-dependent association of common variants of microcephaly genes with brain structure. Proc Natl Acad Sci USA 2010; 107: 384-388.

48. Kaindl AM, Passemard S, Kumar P, Kraemer N, Issa L, Zwirner A et al. Many roads lead to primary autosomal recessive microcephaly. Prog Neurobiol 2010; 90: 363-383.

49. Kirkpatrick B, Buchanan RW, Ross DE, Carpenter WT, Jr. A separate disease within the syndrome of schizophrenia. Arch Gen Psychiatry 2001; 58: 165-171.

50. Crow TJ. Molecular pathology of schizophrenia: more than one disease process? Br Med 1980; 280: 66-68.

51. Marangi G, Ricciardi S, Orteschi D, Lattante S, Murdolo M, Dallapiccola B et al. The PittHopkins syndrome: report of 16 new patients and clinical diagnostic criteria. Am J Med Genet A 2011; 155A: 1536-1545.

52. Emsley R, Chiliza B, Schoeman R. Predictors of long-term outcome in schizophrenia. Curr Opin Psychiatry 2008; 21: 173-177.

53. Sullivan PF, Fan $\mathrm{C}$, Perou $\mathrm{CM}$. Evaluating the comparability of gene expression in blood and brain. Am J Med Genet B Neuropsychiatr Genet 2006; 141B: 261-268.

54. Mudge J, Miller NA, Khrebtukova I, Lindquist IE, May GD, Huntley JJ et al. Genomic convergence analysis of schizophrenia: mRNA sequencing reveals altered synaptic vesicular transport in post-mortem cerebellum. PLOS One 2008; 3: e3625.

55. Bowden NA, Weidenhofer J, Scott RJ, Schall U, Todd J, Michie PT et al. Preliminary investigation of gene expression profiles in peripheral blood lymphocytes in schizophrenia. Schizophr Res 2006; 82: 175-183.

56. Glatt SJ, Everall IP, Kremen WS, Corbeil J, Sasik R, Khanlou N et al. Comparative gene expression analysis of blood and brain provides concurrent validation of SELENBP1 up-regulation in schizophrenia. Proc Natl Acad Sci USA 2005; 102: 15533-15538.

57. Kakiuchi C, Ishiwata M, Nanko S, Ozaki N, Iwata N, Umekage T et al. Up-regulation of ADM and SEPX1 in the lymphoblastoid cells of patients in monozygotic twins discordant for schizophrenia. Am J Med Genet B Neuropsychiatr Genet 2008; 147B: 557-564.

58. Kuzman MR, Medved V, Terzic J, Krainc D. Genome-wide expression analysis of peripheral blood identifies candidate biomarkers for schizophrenia. J Psychiatr Res 2009; 43: 1073-1077.

59. Middleton FA, Pato CN, Gentile KL, McGann L, Brown AM, Trauzzi M et al Gene expression analysis of peripheral blood leukocytes from discordant sib-pairs with schizophrenia and bipolar disorder reveals points of convergence between genetic and functional genomic approaches. Am J Med Genet B Neuropsychiatr Genet 2005; 136B: $12-25$.

60. Numata S, Ueno S, Iga J, Yamauchi K, Hongwei S, Hashimoto R et al. Gene expression in the peripheral leukocytes and association analysis of PDLIM5 gene in schizophrenia. Neurosci Lett 2007; 415: 28-33.

61. Tsuang MT, Nossova N, Yager T, Tsuang MM, Guo SC, Shyu KG et al. Assessing the validity of blood-based gene expression profiles for the classification of schizophrenia and bipolar disorder: a preliminary report. Am J Med Genet B Neuropsychiatr Genet 2005; 133B: $1-5$.

62. Vawter MP, Ferran E, Galke B, Cooper K, Bunney WE, Byerley W. Microarray screening of lymphocyte gene expression differences in a multiplex schizophrenia pedigree. Schizophr Res 2004; 67: 41-52.

63. Zvara A, Szekeres G, Janka Z, Kelemen JZ, Cimmer C, Santha M et al. Over-expression of dopamine D2 receptor and inwardly rectifying potassium channel genes in drug-naive schizophrenic peripheral blood lymphocytes as potential diagnostic markers. Dis Markers 2005; 21: 61-69.

64. Achiron A, Gurevich M. Peripheral blood gene expression signature mirrors central nervous system disease: the model of multiple sclerosis. Autoimmun Rev 2006; 5: 517-522.

65. Williams HJ, Moskvina V, Smith RL, Dwyer S, Russo G, Owen MJ et al. Association between TCF4 and schizophrenia does not exert its effect by common nonsynonymous variation or by influencing cis-acting regulation of $\mathrm{mRNA}$ expression in adult human brain. Am J Med Genet B Neuropsychiatr Genet 2011; 156: 781-784.

66. Buonocore F, Hill MJ, Campbell CD, Oladimeji PB, Jeffries AR, Troakes $C$ et al. Effects of cis-regulatory variation differ across regions of the adult human brain. Hum Mol Genet 2010; 19: 4490-4496.

67. Colantuoni C, Lipska BK, Ye T, Hyde TM, Tao R, Leek JT et al. Temporal dynamics and genetic control of transcription in the human prefrontal cortex. Nature 2011; 478: 519-523.

68. Cho EA, Dressler GR. TCF-4 binds beta-catenin and is expressed in distinct regions of the embryonic brain and limbs. Mech Dev 1998; 77: 9-18.

Translational Psychiatry is an open-access journal published by Nature Publishing Group. This work is licensed under the Creative Commons Attribution-Noncommercial-No Derivative Works 3.0 Unported License. To view a copy of this license, visit http://creativecommons.org/licenses/by-nc-nd/3.0/

\section{Supplementary Information accompanies the paper on the Translational Psychiatry website (http://www.nature.com/tp)}

